\title{
The influence of skeletal muscle anisotropy on electroporation: in vivo study and numerical modeling
}

\author{
Selma Čorović $\cdot$ Anže Županič • Simona Kranjc $\cdot$ \\ Bassim Al Sakere • Anne Leroy-Willig • \\ Lluis M. Mir • Damijan Miklavčič
}

Received: 6 October 2009/Accepted: 11 April 2010/Published online: 28 April 2010

(C) The Author(s) 2010. This article is published with open access at Springerlink.com

\begin{abstract}
The aim of this study was to theoretically and experimentally investigate electroporation of mouse tibialis cranialis and to determine the reversible electroporation threshold values needed for parallel and perpendicular orientation of the applied electric field with respect to the muscle fibers. Our study was based on local electric field calculated with three-dimensional realistic numerical models, that we built, and in vivo visualization of electroporated muscle tissue. We established that electroporation of muscle cells in tissue depends on the orientation of the applied electric field; the local electric field threshold values were determined (pulse parameters: $8 \times 100 \mu \mathrm{s}$, $1 \mathrm{~Hz}$ ) to be $80 \mathrm{~V} / \mathrm{cm}$ and $200 \mathrm{~V} / \mathrm{cm}$ for parallel and perpendicular orientation, respectively. Our results could be useful electric field parameters in the control of skeletal muscle electroporation, which can be used in treatment planning of electroporation based therapies such as gene therapy, genetic vaccination, and electrochemotherapy.
\end{abstract}

S. Čorović · A. Županič · D. Miklavčič $(\varangle)$

Faculty of Electrical Engineering, University of Ljubljana,

Trzaska 25, SI-1000 Ljubljana, Slovenia

e-mail: damijan.miklavcic@fe.uni-lj.si

S. Čorović · B. Al Sakere · L. M. Mir

Institute Gustave-Roussy, CNRS, UMR 8121, 39 Rue C.

Desmoulins, 94805 Villejuif, France

S. Čorović · B. Al Sakere - L. M. Mir

Univ Paris-Sud, UMR 8121, Paris, France

S. Kranjc

Institute of Oncology, Zaloška 2, SI-1000 Ljubljana, Slovenia

A. Leroy-Willig

Univ Paris-Sud, UMR 8081, U2R2M, Batiment 220, 91405

Orsay Cedex, France
Keywords In vivo electroporation - Skeletal muscle . Tissue anisotropy $\cdot$ Magnetic resonance imaging . Local electric field distribution

\section{Introduction}

In vivo electroporation (also termed electropermeabilization) is an effective method for administration of therapeutic drugs (such as chemotherapeutic agents) and one of the most efficient and simple nonviral methods for gene transfer into target tissue, provided appropriate electrical parameters are chosen $[19,21,22]$. In vivo electroporation is successfully applied in clinics for electrochemotherapy of solid tumors [16]. Gene transfer by means of electroporation (gene electrotransfer) has also been performed in humans and it seems likely it could be applied clinically for nonviral gene therapy. Efficient in vivo gene electrotransfer has been shown in a wide range of tissues [2, 4, 27, 33]. Skeletal muscle is one of the most attractive tissues for administration of therapeutic genes by electroporation for both local and systemic gene therapy, for genetic vaccination against infectious agents (e.g. hepatitis B virus, human immunodeficiency virus-1) as well as for basic research of muscle physiology, due to a number of its biological properties such as relatively easy access the skeletal muscles, long term stable transgene expression and excellent vascularisation [13, 17, 26, 35].

In order to assure optimal conditions for gene therapy and genetic vaccination, the electrical parameters (such as applied voltage, electrode shape, and position) need to be chosen to assure reversible muscle electroporation just above the reversible threshold value. After the application of electric pulses the electroporated cell membrane needs to be resealed in order to obtain efficient DNA transfer. 
This can be assured with appropriate pulse parameters that induce reversible muscle electroporation, otherwise the viability of the target muscle fibers is lost, due to the irreversible membrane electroporation (i.e. leading to cell death). The reversible electroporation is required also in electrochemotherapy, so as to avoid exposure of healthy tissues to high electric field (above irreversible threshold) and possible ulceration and wound appearance.

Electroporation pulses establish a local electric field $(E)$ within the treated tissue, which depends on the amplitude of the applied electroporation pulses, electrodes' shape and placement, and tissue geometrical and physical properties $[18,24]$. In order to obtain reversible tissue electroporation, the target tissue has to be subjected to the local electric field inducing reversible electroporation ( $E$ between reversible and irreversible electroporation thresholds $E_{\text {rev }}<E<E_{\text {irrev }}$ ) [19], while the magnitude of $E$ above $E_{\text {irrev }}$ induces irreversible tissue electroporation [29].

By combination of numerical modeling and optimization algorithms, the level and extent of the tissue electroporation can be controlled by appropriate amplitude of electroporation pulses and electrodes shape and placement, provided that the electroporation thresholds, $E_{\text {rev }}$ and $E_{\text {irrev }}$, for each tissue type are known $[5,31,38]$. In a study on liver tissue electroporation, Miklavcic et al. [19] showed that electroporation thresholds of local electric field and electroporated-tissue extent can be efficiently determined by comparing experimental observations (i.e. visualization of electroporated tissue region) to the local electric field distribution obtained by three-dimensional numerical simulations on realistic mathematical models that take into account electric properties of the involved tissues. Since muscle tissue exhibits anisotropic electric properties, its sensitivity to electroporation is expected to be electric field orientation dependent. Thus, determination of electroporation thresholds for different electric field orientations with respect to the muscle fibers is of great importance for optimization of local electric field distribution and for control of electroporation process in the target tissue. Most of in vivo studies on skeletal muscle electroporation have been done for the direction of the electric field perpendicular to the long axis of the fibers [3, 7, 10, 11, 14, 25]. Only, Aihara and Miyazaki [1] experimentally examined also the influence of parallel electric field orientation on gene electrotransfer in skeletal muscle using two needle electrodes. Analytical solution for the electric potential and activating function in two dimensions established by needle electrodes in anisotropic tissue was reported by [12].

Our study was based on the comparison of local electric field calculated using three-dimensional realistic numerical models and in vivo visualization of electroporated target tissue, which for skeletal muscle tissue has not been performed before. The aim of our study was to (1) investigate electroporation of mouse tibialis for parallel and perpendicular orientation of the applied electric field with respect to the muscle fibers and (2) determine the electroporation thresholds of mouse tibialis cranialis muscle using both electric field orientations; theoretically and experimentally using two plate electrodes.

\section{Methods}

\subsection{Animals}

Female C57B1/6 mice were housed and handled according to recommended guidelines [34] and French legislation concerning animal welfare. Prior to all procedures, mice were anesthetized by administrating $12.5 \mathrm{mg} / \mathrm{kg}$ xylazine (Bayer Pharma, Puteaux, France) and $125 \mathrm{mg} / \mathrm{kg}$ ketamine (Parke Davis, France) by intraperitoneal injection in $150 \mu \mathrm{l}$ saline.

\subsection{Electroporation protocol and electrodes}

Electroporation was performed by applying eight rectangular monophasic $100 \mu$ s electroporation pulses at a repetition frequency of $1 \mathrm{~Hz}$. The electroporation pulses were delivered through two stainless-steel parallel plate electrodes (10 mm long and $0.7 \mathrm{~mm}$ wide), which were in direct contact with the tibialis muscle after skin incision (Fig. 1). We carefully controlled the electrode placement by marking its position on the muscle and contact surface formed between the electrode and muscle surface (Fig. 1). Namely, we carefully measured the position of electrodes on the muscle during the in vivo experiments. Those measurements were then taken into account when modeling the geometry of the muscle and the contact surface between the electrodes and the muscle. We aligned the images obtained in in vivo measurements with the images from numerical models by using graphical software (i.e. CorelDraw). Electrode tissue set-ups were examined for perpendicular and parallel orientation of electric field with respect to the long axis of the muscle fibers, as illustrated in Fig. 1a, b, respectively.

Electroporation pulses of amplitude $U<60 \mathrm{~V}$ were generated by a PS 15 electropulsator (Jouan, St. Herblin, France), while the pulses of amplitude $U \geq 60 \mathrm{~V}$ were generated by a Cliniporator (Igea, Carpi, Italy).

\subsection{Magnetic resonance imaging (MRI) experiments}

For the detection of the electroporated muscle volume by means of MRI we used Gd-DOTA (Dotarem, Guerbet, Aulnay-sous-Bois, France), contrast agent. The assessment of reversibly electroporated muscle volume was based on 



Fig. 1 Experimental electrode tissue set-up for a perpendicular and $\mathbf{b}$ parallel orientation of electric field with respect to the long axis of muscle fibers and the corresponding three-dimensional finite element model geometries for c perpendicular and $\mathbf{d}$ parallel electric field orientation. The corresponding contact surfaces between the electrodes and muscle model are depicted below the three-dimensional models in a and $\mathbf{d}$ for perpendicular and parallel orientation, respectively

$\mathrm{T} 1$ relaxation time weighted images. The $\mathrm{T} 1$ weighted images were expected to show the muscle areas where the Gd-DOTA molecules were trapped inside the reversibly electroporated muscle cells. T2 relaxation time weighted images were used to detect the irreversibly electroporated muscle areas by detecting the signs related to the cell damage, such as leakage of Gd-DOTA molecules out of the cells and edema [14] (in the absence of these signs the images showed reversible muscle electroporation).

Twenty mice were anesthetised and Gd-DOTA was injected intraperitoneally at the dose $10 \mathrm{ml} / \mathrm{kg}$ of a diluted solution isoosmotic to plasma ( $1 \mathrm{ml}$ of Dotarem and $2.77 \mathrm{ml}$ of water). It was previously demonstrated that after slow transperitoneal passage to the blood flow, the contrast agent remained in the extracellular space at a roughly constant concentration between 15 and 120 min after the injection [23]. In our experiments, skin was incised and electroporation pulses were delivered directly to the muscle (Fig. 1) $20 \mathrm{~min}$ after the injection.

The amplitudes of the in vivo delivered electroporation pulses were selected based on a preliminary numerical analysis of electric field orientation with respect to the muscle fibers. For the perpendicular orientation of applied electric field (Fig. 1a) 18 muscles were treated with electroporation pulses ranging from 30 to $164 \mathrm{~V}$ (voltage to electrodes distance ratio ranging from 150 to $820 \mathrm{~V} / \mathrm{cm}$ ).
For the parallel orientation (Fig. 1b), 20 muscles were treated with electroporation pulses ranging from 8 to $160 \mathrm{~V}$ (voltage to electrodes distance ratio ranging from 20 to $400 \mathrm{~V} / \mathrm{cm})$. Distances between electrodes $(d)$ were $d=2 \mathrm{~mm}$ for perpendicular (Fig. 1a) and $d=4 \mathrm{~mm}$ for parallel orientation (Fig. 1b). Two injected muscles were not electroporated and served as controls.

After electroporation, skin incisions were sewed up. The signal increase corresponding to the uptake of contrast agent by the reversibly electroporated cells was measured by MRI 3 days later, since complete elimination of the contrast agent located within the extracellular space is reached at day 3 after the injection [23].

\subsubsection{MRI acquisition}

MRI examinations were performed using a 4.7 Tesla system equipped with a Tecmag spectrometer with a home built bird-cage coil (inner diameter $32 \mathrm{~mm}$ ). Mice were positioned with legs extended inside a cylindrical holder fitting inside the coil.

T1-weighted magnetic resonance images were obtained using a T1-weighted spin-echo sequence with the following parameters: TR $644 \mathrm{~ms}$, TE $7.1 \mathrm{~ms}$, spectral width $80 \mathrm{kHz}$, 4 accumulations, in-plane spatial resolution $120 \mu \mathrm{m} \times 150 \mu \mathrm{m}$, slice thickness $2 \mathrm{~mm}$, and slice spacing $2 \mathrm{~mm}$. Eleven successive axial slices perpendicular to the long leg axis and thus to the muscle fibers (i.e. MR images of $Y Z$ cross-sections (Fig. 1)) were obtained in $11 \mathrm{~min}$.

Figure 2 shows T1-weighted images of mice legs treated at voltages producing $E$ above the reversible threshold values (Fig. 2a for perpendicular and Fig. 2b for parallel orientation) and below the reversible thresholds (Fig. 2c for perpendicular and Fig. 2d for parallel orientation). The dotted white lines in Fig. 2 mark the corresponding electrode positions on the treated mice legs. The zones of increased muscle signal (i.e. electroporated muscle regions, where the contrast agent remained inside cells 3 days after the electroporation increased the muscle water signal by shortening its relaxation time T1) are indicated by arrows in Fig. 2a, b.

T2-weighted MR images were obtained using a T2weighted spin-echo sequence with the following parameters: TR $2250 \mathrm{~ms}$ TE $50 \mathrm{~ms}$, two averages, in-plane resolution $400 \mu \mathrm{m} \times 400 \mu \mathrm{m}$, and slice thickness $2 \mathrm{~mm}$. Five successive axial slices perpendicular to the muscle fibers (i.e. MR images of $Y Z$ cross-sections (Fig. 1)) were obtained in $10 \mathrm{~min}$.

Images were reconstructed with zero-filling to improve in plane spatial resolution and analyzed using a home written algorithm (MATLAB 2007a, The MathWorks, Natick, USA). 




Fig. 2 T1-weighted MRI images of four examined mice legs in $Z Y$ cross-section: a $U=101 \mathrm{~V}$, perpendicular, b $U=40 \mathrm{~V}$, parallel, c $U=30 \mathrm{~V}$, perpendicular, and d $U=15 \mathrm{~V}$, parallel orientation. a and $\mathbf{b}$ show the successfully electroporated muscles where the zones of increased muscle signal (electroporated region EP) are indicated by arrows. $\mathbf{c}$ and $\mathbf{d}$ show the muscles with the $E$ below the reversible

\subsubsection{MRI image analysis}

All slices (i.e. MRI images) where a zone of increased signal was detected were taken into account for the analysis (electroporation volume detection). The number of analyzed slices for the perpendicular orientation was $1(2 \mathrm{~mm}$ leg extent at lower voltages applied) to 5 (10 $\mathrm{mm}$, which corresponds to the electrode length). The number of slices analyzed for the parallel orientation was $1(2 \mathrm{~mm})$ to $3(6 \mathrm{~mm}$ leg extent-distance between the electrodes was $4 \mathrm{~mm}$ ). The area with increased signal indicating the successfully electroporated area of tibialis cranialis muscle was analyzed by determination of two regions of interest $\left(\mathrm{ROI}-\mathrm{ROI}_{1}\right.$ and $\mathrm{ROI}_{2}$, as marked in Fig. 3) in each magnetic resonance image obtained. Figure 3 shows an example of the marked ROI in the MRI image obtained with $U=101 \mathrm{~V}$ (the corresponding position of electrodes is marked in Fig. 2a).

The first region of interest $\left(\mathrm{ROI}_{1}\right)$, as marked in Fig. 3 with dotted black line, was carefully drawn by an expert around the zone with increased signal indicating the successfully electroporated area of the tibialis cranialis muscle, after windowing the image to increase the contrast. The number of pixels within the drawn $\mathrm{ROI}_{1}$ region in each of the magnetic resonance images was determined and the obtained sum of the pixels from all magnetic resonance images was converted to the labeled volume by its multiplication with the elementary volume corresponding to one pixel $V=9.010^{-3} \mathrm{~mm}^{3}$. electroporation threshold $\left(E<E_{\mathrm{rev}}\right)$. The electrode placements are illustrated with dashed squares. Distances between electrodes for perpendicular and parallel orientations are 2 and $4 \mathrm{~mm}$, respectively. In $\mathbf{b}$ and $\mathbf{d}$ (parallel orientation); the box corresponds to the position of the two electrodes, one lying $2 \mathrm{~mm}$ above the image and the other $2 \mathrm{~mm}$ below



Fig. 3 T1-weighted MRI image of examined mice leg in $Z Y$ crosssection obtained at applied $U=101 \mathrm{~V}$ in perpendicular orientation. Regions of interest $\mathrm{ROI}_{1}$ is marked with dotted black line. Region of interest $\mathrm{ROI}_{2}$ is marked with solid black line. The corresponding electrode position for this experiment is shown in Fig. 2a

The second region of interest $\mathrm{ROI}_{2}$ (as marked in Fig. 3 with solid black line), slightly larger than the previous one, was manually drawn around the $\mathrm{ROI}_{1}$ in each of the 
magnetic resonance images. The mean signal, $S$, inside this zone was computed. A reference zone, where muscle was not affected by electroporation pulses, was drawn in another unaffected muscle and its mean reference signal, $S_{0 \text {, was computed. }}$

In the larger zone with increased signal $\left(\mathrm{ROI}_{2}\right)$, the signal of each pixel $(i, j)$ is $S(i, j)$ and $S_{0}$ is the estimation of the signal $S_{O}(i, j)$ that would correspond to the local value in the absence of modification due to electroporation.

An index of integrated muscle signal increase was determined from the following relation (Eq. 1):

$I=\sum i, j\left(\left(S(i, j)-S_{0}(i, j)\right) / S_{0}(i, j)\right)$

The determined index, $I$, is robust compared to the delimitation of the ROI where the signal increase is determined, since the same sum is obtained in a larger region of interest, $\mathrm{ROI}_{2}$, than in a closely drawn $\mathrm{ROI}_{1}$. The index, $I$, evaluates the sum of local signal increase that is related to the total amount of contrast agent inside muscle cells as long as the relation between contrast agent concentration and signal is linear [14], which is the case at the dose injected in our experiments.

\subsection{Propidium iodide (PI) uptake experiments}

In order to visualize the extent of muscle electroporation in $X Y$ cross sections of the examined muscles (Fig. 1), a fluorescent dye, PI, (Sigma, Saint Louis, USA) was used. The dye is essentially membrane impermeant, but can enter the cells being electroporated. Because its fluorescence increases several fold upon binding to DNA or mRNA, it can be used to detect cell electroporation within the treated tissue [28].

Eleven mice were anesthetized, the skin facing the muscle was removed and $20 \mu \mathrm{l}(10 \mathrm{mM})$ of PI was slowly injected into the tibialis cranialis (injection duration $=30 \mathrm{~s}$ ). The muscle was electroporated immediately after the injection. The amplitudes of the electroporation pulses delivered to the examined muscles were selected based on a preliminary numerical analysis of electric field orientation with respect to the muscle fibers. The muscle electroporation was investigated for pulse amplitudes $U=50,60,70,80$, and $100 \mathrm{~V}(U / d=250,300,350,400$, and $500 \mathrm{~V} / \mathrm{cm}$ ) for perpendicular orientation and $U=10$, $20,30,40,50 \mathrm{~V}(U / d=50,100,150,200,250$, and $300 \mathrm{~V} / \mathrm{cm}$ ) for the parallel orientation. In order to assess the reproducibility of the fluorescence patterns we repeated the measurements 2-3 times for each of the voltages applied. Two injected muscles were not electroporated and served as controls. Distance between electrodes $(d)$ was $2 \mathrm{~mm}$ for both perpendicular (Fig. 1a) and parallel orientation (Fig. 1b). The PI fluorescence was observed under the stereomicroscope (Leica MZFLIII, Germany) and photographed with a digital camera (Olympus ${ }^{\circledR}$ Camedia C-5050 Zoom) $15 \mathrm{~min}$ after the electroporation. The obtained PI fluorescence displayed the total sum of the signal obtained within the entire muscle observed under the microscope (from the top view). Mice were killed after the experiment while still anesthetized.

\subsection{Numerical modeling}

The three-dimensional model of muscle tissue is based on the numerical solution of partial differential equation for steady electric current in anisotropic conductive media, Eq. 2.

$\nabla \cdot(\sigma \cdot(-\nabla u))=0$,

where $\sigma$ is conductivity tensor $[\mathrm{S} / \mathrm{m}]$ describing anisotropic electric properties of the muscle (Eq. 3), and the negative gradient of the potential $u[\mathrm{~V}]$ in the tissue volume defines vector of electric field intensity $\vec{E}[\mathrm{~V} / \mathrm{m}]$.

The numerical calculations were performed by means of finite element method using COMSOL Multiphysics 3.4 software package in the 3D Conductive Media DC application mode on a PC running Windows XP with a 3.00 GHz Pentium D processor and $2 \mathrm{~GB}$ of RAM.

\subsubsection{Model geometry and electric properties of muscle tissue}

In our numerical model, the muscle tissue geometry is represented as an ellipsoid (Fig. 1c, d), with radii $8 \mathrm{~mm}$ and $2.4 \mathrm{~mm}$ along $X$ - and $Y$-axis, respectively, and the radius $2 \mathrm{~mm}$ along $Z$-axis. The model is oriented in the orthogonal Cartesian system so that the long axis of the muscle fibers is aligned with the axis $X$. The contact surfaces between the electrode and muscle were modeled to be as similar as possible to the contact surfaces obtained in in vivo experiments.

\subsubsection{Electric properties of the modeled tissues and electroporation process modeling}

Before applying electroporation pulses or if the amplitude of the applied electroporation pulses was too low to produce the local electric field above the reversible electroporation threshold $\left(E<E_{\mathrm{rev}}\right)$, the muscle was modeled with constant electric conductivity $\sigma[\mathrm{S} / \mathrm{m}]$. The muscle tissue was considered anisotropic, having higher conductivity along the muscle fibers $\left(\sigma_{x x}=0.75 \mathrm{~S} / \mathrm{m}\right)$ compared to the conductivities perpendicular to the fibers $\left(\sigma_{y y}=\right.$ $\left.\sigma_{z z}=0.135 \mathrm{~S} / \mathrm{m}\right)$, as represented with a diagonal conductivity matrix, Eq. 3 . 
$\sigma=\left[\begin{array}{ccc}\sigma_{x x} & 0 & 0 \\ 0 & \sigma_{y y} & 0 \\ 0 & 0 & \sigma_{z z}\end{array}\right]$.

These values were selected considering both our previous numerical and in vivo electroporation studies [7, 25] and the measurements of muscle tissue conductivity found in the available literature [20].

If the local electric field in the tissues exceeded the value $E_{\text {rev }}\left(E>E_{\text {rev }}\right)$ the tissue conductivity changed according to the function $\sigma(E)$. Namely, the dynamics of the electroporation process (i.e. the conductivity changes during the eight pulses) in the treated tissue was modeled with a sequence static finite element models according to a sequential permeabilization model, proposed by Sel et al. [30]. In each step in sequence (i.e. static finite element model $k$ ) the tissue conductivity was determined based on electric field distribution calculated in the previous step in sequence (i.e. static finite element model $k-1$ ), as described in equation Eq. 4

$\sigma(k, E)=f(E(k-1))$,

where $\sigma$ is electric conductivity of the modeled tissue, $k$ is number of static finite element models in sequence, and $E$ is local electric field distribution calculated in each of the steps $k$. In our models the $\sigma(E)$ function was considered to be sigmoid and electric conductivity at the end of the pulse delivery increased by a factor of 3.5 in parallel direction and by a factor of 0.135 in perpendicular direction compared to the initial electric conductivity values $\left(\sigma_{x x}\right.$ and $\sigma_{y y}=\sigma_{z z}$, respectively). The sigmoid shape of the $\sigma(E)$ function and the electric conductivity values at the end of the pulse delivery were determined based on comparison of the electric current measured in vivo and the electric current calculated in realistic numerical model in our previous study (Corovic et al., 2010, submitted to Comptes Rendus Physiques).

\subsubsection{Boundary conditions and mesh parameters}

Dirichlet boundary condition was defined by applying constant voltages, $U$, between the electrodes. The Neumann boundary condition, i.e., the insulation condition, was set to the rest of the outer boundaries of the model. The electric field distribution was calculated for each of the applied voltage. The results of numerical simulations were controlled by refining the mesh until the difference in numerical solutions was negligible (less than $0.5 \%$ ) when the number of finite elements (i.e. mesh density) increased. The resulting three-dimensional finite element muscle models illustrated in Fig. 1c, d, consisted of 90,213 and 89,420 elements, respectively.

\subsubsection{Analysis of the local electric field distribution}

Experimentally determined reversible thresholds $E_{\mathrm{rev} \|}=$ $80 \mathrm{~V} / \mathrm{cm}$ and $E_{\mathrm{rev} \perp}=200 \mathrm{~V} / \mathrm{cm}$ for parallel and perpendicular electric field orientation, respectively, were included in the numerical muscle models (Fig. 1c, d) to calculate the volume $(V)$ of the muscle tissue exposed to the local electric field above the thresholds. The calculations of $V$ were performed with an algorithm, which was written in MATLAB 2007a and run together with the numerical calculation using the link between MATLAB and COMSOL. The numerically calculated volumes for each of the applied voltages for parallel and perpendicular electric field orientation were then compared to the experimentally obtained labeled volumes of the muscles examined by using MRI.

\section{Results}

\subsection{MRI results and numerical calculations}

The experimentally obtained labeled volume that corresponds to the successfully eletroporated area of tibialis cranialis muscle (i.e. the detection of signal increase in T1-weighted images), obtained in all examined extremities with electric fields parallel and perpendicular to the long axis of muscle fibers are shown in Fig. 4a. The labeled volume is negligible at low voltages applied $U /$ $d \leq 75 \mathrm{~V} / \mathrm{cm}$ for parallel orientation and $U / d \leq 195 \mathrm{~V} /$ $\mathrm{cm}$ for perpendicular orientation. The comparison of labeled volume determined in vivo and numerically calculated volume above reversible threshold values for parallel and perpendicular orientations is shown in Fig. 4b, c, respectively. General agreement was obtained. Based on this the electric field threshold values $E_{\mathrm{rev} \|}$ and $E_{\mathrm{rev} \perp}$ were determined to be $80 \mathrm{~V} / \mathrm{cm}$ and $200 \mathrm{~V} / \mathrm{cm}$ for parallel and perpendicular orientation, respectively. The labeled volume in Fig. 4 exhibits a plateau at about $35 \mathrm{~mm}^{3}$ both with perpendicular electric field (from 250$300 \mathrm{~V} / \mathrm{cm}$ to $800 \mathrm{~V} / \mathrm{cm}$ ), and with the parallel electric field (from 200 to $400 \mathrm{~V} / \mathrm{cm}$ ).

The detection of signal increase in T2-weighted images showed muscle edema in two legs (at $800 \mathrm{~V} / \mathrm{cm}$, perpendicular orientation, data not shown), which corresponds to the irreversibly electroporated muscle, as previously demonstrated [23]. The muscle edema was not observed at electric field lower than $800 \mathrm{~V} / \mathrm{cm}$ (which indicated that at $U / d<800 \mathrm{~V} / \mathrm{cm}$ the treated muscle tissue was not irreversibly electroporated).

Figure 5 shows the index of signal increase inside the tibialis cranialis muscle obtained in all examined 



Fig. 4 In vivo MRI determined labeled volume within the tibialis cranialis muscle, determined in all examined extremities with parallel and perpendicular electric field (a) and the comparison of labeled volume determined in vivo and numerically calculated volume above reversible threshold values for parallel (b) and perpendicular electric field orientation (c)

extremities for parallel and perpendicular electric field. This index, defined by Eq. 1, is roughly proportional to the quantity of contrast agent inside the treated zone. In contrast to the labeled volumes in Fig. 4, however, the index of signal increase in Fig. 5 does not exhibit the plateau but continues to increase with the applied voltage $U$, except at the highest value of $U(U / d=800 \mathrm{~V} / \mathrm{cm})$ where the signal decreases.

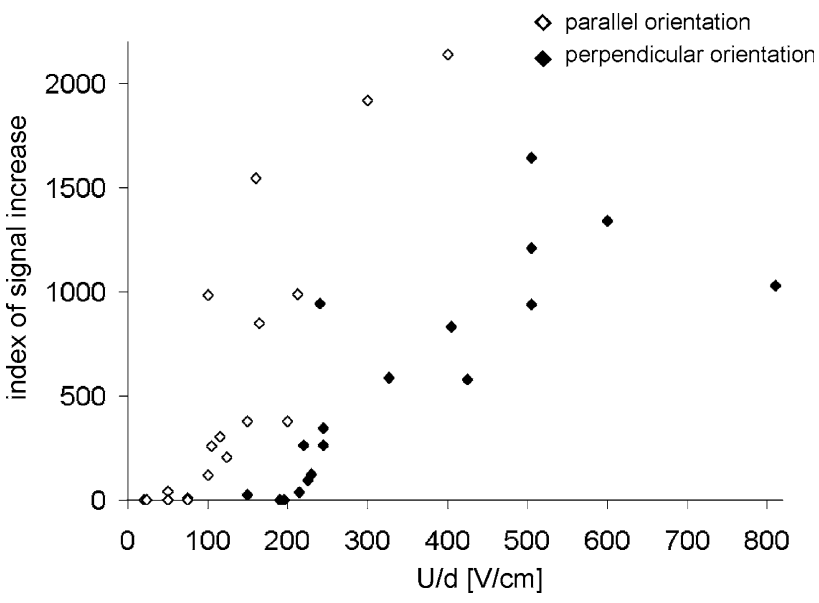

Fig. 5 Index of MRI signal increase within the tibialis cranialis muscle obtained in all examined extremities for parallel and perpendicular electric field

\subsection{PI fluorescence and numerical modeling}

\subsubsection{Parallel orientation of the electric field vs. muscle fibers}

Experimentally visualized PI fluorescence within the parallelly electroporated muscles for the applied $U=10,20$, 30 , and $40 \mathrm{~V}$ (i.e. $U / d=50,100,150$, and $200 \mathrm{~V} / \mathrm{cm}$ ) is shown in Fig. 5a). The corresponding electric field distribution calculated at the beginning (step 1 of sequence analysis) and at the end (step 5 of sequence analysis) of each of the voltage pulses of the same amplitude as applied in in vivo experiments is shown in Fig. 6b, c. The reversible electroporation threshold value $E_{\text {rev } \|}=80 \mathrm{~V} / \mathrm{cm}$, previously determined experimentally by means of MRI, was included in the numerical muscle model as a parameter of sigmoid $\sigma(E)$ function for all the voltages applied. The electric field is displayed in the range from the reversible threshold value $E_{\text {rev } \|}=80$ to $800 \mathrm{~V} / \mathrm{cm}$. In order to compare the shapes of experimentally and numerically detected electroporated muscle regions, the numerically obtained contour of local electric field strength, corresponding to the reversible electroporation threshold $\left(E_{\mathrm{rev} \|}=80 \mathrm{~V} / \mathrm{cm}\right)$, calculated at the end of permeabilization (Fig. 6c), was added to the experimentally visualized images (white solid line in Fig. 6a). The dotted white lines in Fig. 6 correspond to the contact line formed between the electrodes and muscle surface.

\subsubsection{Perpendicular orientation of the electric field versus muscle fibers}

Experimentally visualized PI fluorescence within the perpendicularly electroporated muscles for the applied 
Fig. 6 Measured propidium iodide fluorescence of in vivo electroporated tibialis cranialis (a) and local electric field distribution $E$ (displayed in $X Y$ plane $0.3 \mathrm{~mm}$ below electrodes) for applied $U=10,20,30$ and $40 \mathrm{~V}$ (i.e. $U / d=50,100,150$, and $200 \mathrm{~V} / \mathrm{cm}$ ) calculated: b at the beginning of the pulse and $c$ at the end of the pulse. The calculated $E$ is displayed in the range from reversible threshold value $E_{\text {rev } \|}=80$ to $800 \mathrm{~V} / \mathrm{cm}$. The dotted white line corresponds to the contact line formed between the electrodes and muscle surface. The solid white line in a denotes to the contour of local electric field strength, corresponding to the reversible electroporation threshold $\left(E_{\mathrm{rev} \|}=80 \mathrm{~V} / \mathrm{cm}\right)$, calculated at the end of permeabilization. The dotted and solid white lines from the numerical models were aligned with the fluorescence images by using graphical software (i.e. CorelDraw)


$U=60,70,80$, and $100 \mathrm{~V}$ (i.e. $U / d=300,350,400$ and $500 \mathrm{~V} / \mathrm{cm}$ ) is shown in Fig. 7a. The corresponding electric field distribution calculated at the beginning and at the end of each of the voltage pulse, $U$, of the same amplitude as applied in in vivo experiments is shown in Fig. 7b, c. The reversible electroporation threshold value $E_{\mathrm{rev} \perp}=200 \mathrm{~V} /$ $\mathrm{cm}$, previously determined experimentally by means of MRI, was included in the numerical muscle model as a parameter of sigmoid $\sigma(E)$ function for all the voltages applied. The local electric field is also displayed in the range from the reversible threshold value 80 to $800 \mathrm{~V} / \mathrm{cm}$. The contour of electric field strength corresponding to the reversible threshold for perpendicular orientation $\left(E_{\mathrm{rev} \perp}=200 \mathrm{~V} / \mathrm{cm}\right)$ is drawn by a solid white line. The same numerically obtained contour at the end of pulse (Fig. 7c) was added to the experimentally visualized images in order to compare the shapes of experimentally and numerically detected electroporated muscle regions (white solid line in Fig. 7a). The dotted white lines in Fig. 7 correspond to the contact line formed between the electrodes and muscle surface.

The obtained PI fluorescence in Figs. 6a and 7a displays the total sum of the signal obtained within the entire muscle observed under the microscope (from the top view). The local electric field is more pronounced (i.e. the fluorescence is more intense) within the muscle region where the electrodes are more pressed against the muscle due to better contact obtained between the electrodes and the muscle surface. The numerically calculated electric field distribution is displayed in Figs. 6 and 7 in the largest $X Y$ cross section plane of the muscle volume exposed to the $E>E_{\text {rev }}$. The electric field in Figs. $6 \mathrm{~b}$ and $7 \mathrm{~b}$ shows the conditions describing the electric properties of muscle at the beginning of the electroporation process (i.e. first step in sequence analysis) when small changes in tissue conductivity due to the muscle electroporation were expected [25, 30].

From the Figs. $6 \mathrm{~b}$ and $7 \mathrm{~b}$ it can be seen that at the beginning of the pulse the highest local electric field is distributed around the electrodes. In the course of voltage pulse the muscle region exposed to the highest local $E$ is being electroporated first, thus its conductivity increases according to the $\sigma(E)$ function and subsequently reduces the local $E$ in the muscle around the electrodes. During the same voltage pulse applied, as in a voltage divider, the electric field redistributes towards the tissue region with 
Perpendicular orientation



$U=80 \mathrm{~V} \quad U / \mathrm{d}=400 \mathrm{~V} / \mathrm{cm}$



$U=100 \mathrm{~V} U / \mathrm{d}=500 \mathrm{~V} / \mathrm{cm}$






reversible electroporation threshold for perpendicular orientation $\left(E_{\mathrm{rev} \perp}=200 \mathrm{~V} / \mathrm{cm}\right)$ is drawn by a solid white line. The dotted white line corresponds to the contact line formed between the electrodes and muscle surface. The dotted and solid white lines from the numerical models were aligned with the fluorescence images by using graphical software (i.e. CorelDraw) calculated: $\mathbf{b}$ at the beginning of the pulse and $\mathbf{c}$ at the end of pulse. The calculated $E$ is also displayed in the range from 80 to $800 \mathrm{~V} / \mathrm{cm}$. The contour of local electric field strength corresponding to the

lower conductivity in the middle between electrodes [24]. Accordingly, at the end of the pulse we obtained higher electric field in the middle between electrodes and lower electric field in the muscle around electrodes (Figs. 6c, 7c) compared to the electric field distribution obtained at the beginning of the same pulse applied (Figs. 6b, 7b). From the comparison of in vivo experiments and corresponding electric field distribution calculated at the beginning and at the end of pulse we showed that the visualized PI fluorescence corresponding to the in vivo electroporated muscle (Figs. 6a, 7a) comprises both components: the electroporated region obtained at the beginning (Figs. 6b, $7 \mathrm{~b}$ ) and at the end of pulse (Figs. 6c, 7c).

By comparing experimental and numerical results we thus detected successfully electroporated area of the muscle obtained for each of the applied voltages. In this way we also detected the shape of the local electric field distribution above the electroporation thresholds $(E>80 \mathrm{~V} /$ $\mathrm{cm}$ for parallel and $E>200 \mathrm{~V} / \mathrm{cm}$ for perpendicular orientation) inside the muscle for each of the applied voltages. As expected, muscle electroporation was detected at lower voltages for parallel orientation of electric field versus long axis of the muscle fibers $(U=10 \mathrm{~V})$ compared to the perpendicular orientation $(U=60 \mathrm{~V})$.

\section{Discussion}

In our present study, we numerically and experimentally: (1) determined the electroporation thresholds of mouse tibialis cranialis muscle in vivo for parallel and perpendicular orientation of the applied electric field with respect to the muscle fibers (at given pulse parameters, i.e., eight pulses, $100 \mu \mathrm{s}$, frequency $1 \mathrm{~Hz}$ ) and (2) analyzed the difference in successfully electroporated muscle volume and extent using both electric field orientations. Our study was based on the comparison of local electric field calculated with three-dimensional realistic numerical models and in vivo visualization of electroporated target tissue, which for skeletal muscle tissue (mouse tibialis cranialis) has not been performed before.

The numerical calculations were performed using the finite element method, which has proven to be effective in numerical modeling and optimization of electric field distribution in cells and tissues exposed to electric pulses [6, 24]. The in vivo electroporation detection was performed by means of two different in vivo tests: MRI detecting the electrotransfer of a strictly extracellular contrast agent GdDOTA $[14,23]$ and fluorescence visualization of PI binding to DNA or mRNA of the electroporated cells $[9,28]$. 
Both tests allowed for visualization of the level and the extent of muscle electroporation and thus indirect determination of the local electric field distribution in the examined muscles. We used parallel plate electrodes, thus the error of an approximate estimation of local electric field by calculating $U / d$ ratio is small enough since the muscle tissue was electroporated without skin, i.e., only for one type of tissue placed between electrodes [7]. The in vivo results were than compared to the numerical calculations. Good agreement between numerical calculations and experimental observations was obtained (Figs. 4, 6, 7). The agreement between numerically calculated results and experimental observations validated our three-dimensional model. The main purpose of our study was to directly compare the results of numerical calculations to the experimental observations [i.e. muscle volume exposed to the $E>E_{\mathrm{rev}}$ in the model to in vivo electroporated muscle volume detected by MRI (Fig. 4) and by PI fluorescence measurements (Figs. 6, 7]. However, for a detailed statistical analysis more experimental data would be needed.

We demonstrated that the local electric field distribution in muscle tissue strongly depends on the orientation of the electric field with respect to the muscle fibers and thus on electrode placement. A lower magnitude of local electric field was needed to electroporate muscle tissue when the electric field was oriented parallel to the long axis of the muscle fibers compared to the perpendicular orientation, indicating lower electroporation threshold for electric field parallel to the muscle fibers. By direct comparison of calculated electric field distribution with the PI fluorescent regions of the muscle we found that muscle electroporation occurs at lower applied voltage when the electric field is parallel to the muscle fibers for the same distance between electrodes.

The electric field threshold values for the specific pulse parameters $E_{\mathrm{rev} \|}$ and $E_{\mathrm{rev} \perp}$ were determined to be 80 and $200 \mathrm{~V} / \mathrm{cm}$ for parallel and perpendicular orientation of the applied electric field with respect to the long axis of the muscle fibers, respectively (Fig. 2). As the electroporation thresholds depend on pulse characteristics, for other pulse durations and frequencies it will be necessary to determine corresponding threshold values experimentally and include them in the model. It is to be noted that the labeled volume exhibits a plateau at about $35 \mathrm{~mm}^{3}$ both with perpendicular electric field (from $250-300 \mathrm{~V} / \mathrm{cm}$ to $800 \mathrm{~V} / \mathrm{cm}$ ), and with the parallel electric field (from 200 to $400 \mathrm{~V} / \mathrm{cm}$ ) (Fig. 4), while the index of the signal increase in this volume does not show such a plateau (Fig. 5). Actually, the plateau indicates the maximum volume of tissue that can be permeabilized with the electrodes used in our experiments while the integral shows that the level of reversible electroporation of the muscle fibers continues to increase with increasing values of the voltage to electrode distance ratio [except at the highest value $(800 \mathrm{~V} / \mathrm{cm})$, where integral decreases, probably due to the fact that irreversible electroporation occurred]. The plateau is obtained at approximately one half of total mouse tibialis cranialis volume (which is approximately $60 \mathrm{~mm}^{3}$ ). A larger area of the muscle could be treated with different shape and placement of electrodes.

The behavior at $800 \mathrm{~V} / \mathrm{cm}$ is coherent with the occurrence of muscle cell damage that causes the leakage of the contrast agent out of the cells and the edema observed on T2 weighted images [14]. Based on this we can conclude that the irreversible threshold value $E_{\mathrm{irrev} \perp}$ should be near $800 \mathrm{~V} / \mathrm{cm}$ for transversal orientation of $E$ with respect to the long axis of the muscle fiber, while $E_{\text {irrev\| }}$ (for parallel orientation of $E$ ) cannot be determined from the data reported in Fig. 4, but is higher than $400 \mathrm{~V} / \mathrm{cm}$ (i.e. maximum $E$ at which the measurements were done).

The findings of our study on skeletal muscle tissue are in agreement with recently published study on electric field effects on isolated ventricular myocytes by [8], where the myocytes were found to be more sensitive to the electric field when it was applied parallel (versus perpendicular) to the cell major axis. Also, the electroporation threshold values determined in our study are consistent with other studies on muscle tissue electroporation using electric field perpendicular to the long axis of the muscle fibers, either in vivo [7, 23] or in vivo and in silico [25]. The results of our numerical modeling are in agreement also with findings of numerical studies by [32] and [36] demonstrating that the induced transmembrane potential in ellipsoidal cells oriented parallel to the electric field is minimal, while it is maximal for ellipsoidal cells that lie perpendicular to the applied electric field. However, we found the electroporation threshold for perpendicular orientation to be lower compared to the voltage over distance between electrodes ratio in muscle tissue reported by Gehl et al. [11], since in our study the muscle electroporation was experimentally and numerically (in three-dimensional models) examined based on local electric field distribution without presence of the skin. The $E_{\text {rev }}$ in our study is also significantly lower compared to the ratio $U / d(1,000-1,300 \mathrm{~V} / \mathrm{cm})$, which was empirically determined for the electrochemotherapy of cutaneous tumors, due to the fact that the electroporation threshold value depends also on the type of the tissue.

In conclusion, the determined local electric field thresholds of electroporation enable better control of muscle tissue electroporation, and can be used to predict the optimal window for gene electrotransfer, which can facilitate the translation of gene therapy and genetic vaccination into the clinical practice. Results of our study can also be of interest for clinical electrochemotherapy and transdermal drug and gene delivery, since it provides insight into sensitivity of underlying muscle tissue to the 
electroporation procedure [15, 37]. The findings of our study can furthermore significantly contribute to the understanding of electroporation process of other tissues that exhibits anisotropic electric properties.

Acknowledgment This study was supported by the Slovenian Research Agency, CNRS (Centre National de la Recherche Scientifique), IGR, Université Paris-Sud and Ad-Futura.

Open Access This article is distributed under the terms of the Creative Commons Attribution Noncommercial License which permits any noncommercial use, distribution, and reproduction in any medium, provided the original author(s) and source are credited.

\section{References}

1. Aihara H, Miyazaki J (1998) Gene transfer into muscle by electroporation in vivo. Nat Biotechnol 16:867-870

2. Andre F, Gehl J, Sersa G, Preat V, Hojman P, Eriksen J, Golzio M, Cemazar M, Pavselj N, Rols M, Miklavcic D, Neumann E, Teissie J, Mir LM (2008) Efficiency of high- and low-voltage pulse combinations for gene electrotransfer in muscle, liver, tumor, and skin. Hum Gene Ther 19:1261-1271

3. Batiuskaite D, Cukjati D, Mir LM (2003) Comparison of in vivo electropermeabilization of normal and malignant tissue using the ${ }^{51}$ Cr-EDTA uptake test. Biologija 2:45-47

4. Bettan M, Ivanov M, Mir LM, Boissiere F, Delaere P, Scherman D (2000) Efficient DNA electrotransfer into tumors. Bioelectrochemistry 52:83-90

5. Corovic S, Zupanic A, Miklavcic D (2008) Numerical modeling and optimization of electric field distribution in subcutaneous tumor treated with electrochemotherapy using needle electrodes. IEEE Trans Plasma Sci 36:1665-1672

6. Corovic S, Al Sakere B, Haddad V, Miklavcic D, Mir LM (2008) Importance of contact surface between electrodes and treated tissue in electrochemotherapy. Technol Cancer Res Treat 7:393-399

7. Cukjati D, Batiuskaite D, Andre F, Miklavcic D, Mir LM (2007) Real time electroporation control for accurate and safe in vivo non-viral gene therapy. Bioelectrochemistry 70:501-507

8. de Oliveira P, Bassani R, Bassani J (2008) Lethal effect of electric fields on isolated ventricular myocytes. IEEE Trans Biomed Eng 55:2635-2642

9. Gabriel B, Teissie J (1997) Direct observation in the millisecond time range of fluorescent molecule asymmetrical interaction with the electropermeabilized cell membrane. Biophys J 73:2630-2637

10. Gehl J, Mir LM (1999) Determination of optimal parameters for in vivo gene transfer by electroporation, using a rapid in vivo test for cell permeabilization. Biochem Biophys Res Commun 261:377-380

11. Gehl J, Sorensen T, Nielsen K, Raskmark P, Nielsen S, Skovsgaard T, Mir LM (1999) In vivo electroporation of skeletal muscle: threshold, efficacy and relation to electric field distribution. Biochim Biophys Acta 1428:233-240

12. Guo L, Cranford JP, Neu JC, Neu WK (2009) Activating function of needle electrodes in anisotropic tissue. Med Biol Eng Comput 47:1001-1010

13. Hojman P, Zibert J, Gissel H, Eriksen J, Gehl J (2007) Gene expression profiles in skeletal muscle after gene electrotransfer. BMC Mol Biol 8:56

14. Leroy-Willig A, Bureau M, Scherman D, Carlier P (2005) In vivo NMR imaging evaluation of efficiency and toxicity of gene electrotransfer in rat muscle. Gene Ther 12:1434-1443
15. Mali B, Jarm T, Corovic S, Paulin-Kosir M, Cemazar M, Sersa G, Miklavcic D (2008) The effect of electroporation pulses on functioning of the heart. Med Biol Eng Comput 46:745-757

16. Marty M, Sersa G, Garbay J, Gehl J, Collins C, Snoj M, Billard V, Geertsen P, Larkin J, Miklavcic D, Pavlovic I, Paulin-Kosir S, Cemazar M, Morsli N, Rudolf Z, Robert C, O’Sullivan G, Mir LM (2006) Electrochemotherapy —an easy, highly effective and safe treatment of cutaneous and subcutaneous metastases: Results of ESOPE (European Standard Operating Procedures of Electrochemotherapy) study. Eur J Cancer Suppl 4:3-13

17. Mathiesen I (1999) Electropermeabilization of skeletal muscle enhances gene transfer in vivo. Gene Ther 6:508-514

18. Miklavcic D, Beravs K, Semrov D, Cemazar M, Demsar F, Sersa G (1998) The importance of electric field distribution for effective in vivo electroporation of tissues. Biophys J 74:2152-2158

19. Miklavcic D, Semrov D, Mekid H, Mir LM (2000) A validated model of in vivo electric field distribution in tissues for electrochemotherapy and for DNA electrotransfer for gene therapy. Biochim Biophys Acta 1523:73-83

20. Miklavcic D, Pavselj N, Hart XF (2006) Electric properties of tissues. In: Akay M (ed) Wiley encyclopedia of biomedical engineering. Wiley, New York

21. Mir LM (2001) Therapeutic perspectives of in vivo cell electropermeabilization. Bioelectrochemistry 53:1-10

22. Mir LM, Bureau M, Gehl J, Rangara R, Rouy D, Caillaud J, Delaere P, Branellec D, Schwartz B, Scherman D (1999) Highefficiency gene transfer into skeletal muscle mediated by electric pulses. Proc Natl Acad Sci USA 96:4262-4267

23. Paturneau-Jouas M, Parzy E, Vidal G, Carlier P, Wary C, Vilquin J, de Kerviler E, Schwartz K, Leroy-Willig A (2003) Electrotransfer at MR imaging: tool for optimization of gene transfer protocols-feasibility study in mice. Radiology 228: 768-775

24. Pavselj N, Miklavcic D (2008) Numerical modeling in electroporation-based biomedical applications. Radiol Oncol 42:159168

25. Pavselj N, Bregar Z, Cukjati D, Batiuskaite D, Mir LM, Miklavcic D (2005) The course of tissue permeabilization studied on a mathematical model of a subcutaneous tumor in small animals. IEEE Trans Biomed Eng 52:1373-1381

26. Perez N, Bigey P, Scherman D, Danos O, Piechaczyk M, Pelegrin M (2004) Regulatable systemic production of monoclonal antibodies by in vivo muscle electroporation. Genet Vaccines Ther 2:2

27. Prud'homme G, Glinka Y, Khan A, Draghia-Akli R (2006) Electroporation-enhanced nonviral gene transfer for the prevention or treatment of immunological, endocrine and neoplastic diseases. Curr Gene Ther 6:243-273

28. Rols MP, Teissie J (1990) Electropermeabilisation of mammalian cells. Quantitative analysis of the phenomenon. Biophys $\mathrm{J}$ 58:1089-1098

29. Rubinsky J, Onik G, Mikus P, Rubinsky B (2008) Optimal parameters for the destruction of prostate cancer using irreversible electroporation. J Urol 180:2668-2674

30. Sel D, Cukjati D, Batiuskaite D, Slivnik T, Mir LM, Miklavcic D (2005) Sequential finite element model of tissue electropermeabilization. IEEE Trans Biomed Eng 52:816-827

31. Sel D, Macek-Lebar A, Miklavcic D (2007) Feasibility of employing model-based optimization of pulse amplitude and electrode distance for effective tumor electropermeabilization. IEEE Trans Biomed Eng 54:773-781

32. Susil R, Semrov D, Miklavcic D (1998) Electric field-induced transmembrane potential depends on cell density and organization. Electro Magnetobiol 17:391-399

33. Tevz G, Pavlin D, Kamensek U, Kranjc S, Mesojednik S, Coer A, Sersa G, Cemazar M (2008) Gene electrotransfer into murine 
skeletal muscle: a systematic analysis of parameters for long-term gene expression. Technol Cancer Res Treat 7:91-101

34. UKCCCR (1998) Guidelines for welfare of animals in experimental neoplasia (Second Edition). Br J Cancer 77:1-10

35. Umeda Y, Marui T, Matsuno Y, Shirahashi K, Iwata H, Takagi H, Matsumoto K, Nakamura T, Kosugi A, Mori Y, Takemura H (2004) Skeletal muscle targeting in vivo electroporation-mediated HGF gene therapy of bleomycin-induced pulmonary fibrosis in mice. Lab Invest 84:836-844

36. Valic B, Golzio M, Pavlin M, Schatz A, Faurie C, Gabriel B, Teissie J, Rols M, Miklavcic D (2003) Effect of electric field induced transmembrane potential on spheroidal cells: theory and experiment. Eur Biophys J 32:519-528

37. Zupanic A, Ribaric S, Miklavcic D (2007) Increasing the repetition frequency of electric pulse delivery reduces unpleasant sensations that occur in electrochemotherapy. Neoplasma 54:246-250

38. Zupanic A, Corovic S, Miklavcic D (2008) Optimization of electrode position and electric pulse amplitude in electrochemotherapy. Radiol Oncol 42:93-101 